\title{
PROGRESS REPORT ON THE PROJEGT IN RADIO ASTRONOMY AT THE G. R. AGASSIZ STATION OF HARVARD OBSERVATORY
}

\author{
B. J. BOK \\ Harvard College Observatory, Cambridge, Mass., U.S.A.
}

Two years ago I reported at the Jodrell Bank symposium on the initiation of the Agassiz Station project in Radio Astronomy. At that time the 24-ft. antenna was under construction and the electronic equipment for observation of the $2 \mathrm{I}-\mathrm{cm}$. line of neutral hydrogen was being built by Harold I. Ewen, who is the co-director of the project. The first successful observations were obtained in the autumn of 1953 and reported early in 1954 (see Bok [1]). Since then two papers have been published giving the results of the first studies by Heeschen [2] and Lilley [3]; a report on the equipment and the basic programme was given by Bok and Ewen [4]. Earlier this year, at the Princeton Meeting of the American Astronomical Society, Bok [5] drew heavily upon the Agassiz Station results in a paper entitled 'Gas and Dust in Interstellar Clouds', in which an attempt was made to blend the results of radio and optical research. We note also at this point a joint paper by Heeschen and Lilley [6] in which attention was drawn to the important role of Gould's Belt in the distribution of neutral hydrogen in the vicinity of the sun.

\section{INSTRUMENTS*}

For the present our activity is limited entirely to research on the $2 \mathrm{I}-\mathrm{cm}$. line of neutral hydrogen. The receiver built by Ewen has a band-width of I $5 \mathrm{kc}$./s., which at $1420 \mathrm{Mc}$. $/ \mathrm{s}$. is equivalent to a resolution in radial velocity of the order of $3 \mathrm{~km}$. $/ \mathrm{sec}$. The equipment that is presently under construction will have a band-width of $5 \mathrm{kc}$./s., with an equivalent resolution of $\mathrm{I} \mathrm{km}$./sec. in radial velocity. It will have twenty channels, the output of which is recorded by an electrically operated typewriter. It will be

* As no space could be allowed within this symposium publication for description of telescopes and electronic equipment, this section has been greatly condensed. 
used mostly with the 6o-ft. reflector now being built for us by D. S. Kennedy and Company of Cohasset, Massachusetts. This reflector is constructed of welded aluminium tubing covered with extended aluminium mesh measuring $0.95 \mathrm{~cm}$. on a side. The focal ratio is 0.35 and the equatorial mounting will give full hemispherical sky coverage. The expected accuracy of positioning and following is $I^{\prime}$.

\section{OUTLINE OF THE AGASSIZ STATION PROGRAMMES}

From the start, it has been our aim to take the best advantage of the high resolution in frequency of our equipment as is demonstrated by the published papers of Heeschen [2] and Lilley [3] and by the three Harvard papers to follow. The emphasis in our work to date has been primarily on regional surveys as detailed as can be undertaken with our equipment, and on parallel radio and optical research. It is becoming more and more apparent that radio and optical astronomy are not separate or loosely related fields, but rather that the techniques of radio and optical research neatly supplement each other in the study of the physical universe, and notably so for the study of the distribution of hydrogen, neutral and ionized, in our Milky Way system. Heeschen has followed this approach in his study of the $2 \mathrm{I}-\mathrm{cm}$. profiles for fields in the section of Sagittarius and Ophiuchus, and Lilley similarly in his study of the anti-centre section, which established clearly that neutral hydrogen is present in increased amounts in the large complexes of cosmic dust. A subsequent paper by Bok, Lawrence and Menon [7] presented further evidence on the relation between neutral hydrogen and cosmic dust. The $2 \mathrm{I}-\mathrm{cm}$. observations do not indicate a further increase in the amount of neutral atomic hydrogen in the densest dark spots in Taurus and Ophiuchus when the relevant signal strengths are compared with the strengths observed for positions that are less markedly affected by cosmic dust, though still inside the dust complex.

The three studies on which we report in the following papers (8 to ro) are concerned with various aspects of research in the fine structure of the distribution of neutral hydrogen in the Milky Way system. In Matthews' paper, which is a continuation of his paper presented at the Berkeley Meeting of the American Association for the Advancement of Science [8], an attempt is made to unravel some of the details of spiral structure for the section between galactic longitudes $l=60^{\circ}$ and $130^{\circ}$. In his research special emphasis is placed on those directions for which the relevant optical data are established with highest precision. In Menon's research, 
the primary aim is to study the distribution of neutral hydrogen for the Orion nebula and its surroundings; in the hope of establishing a model hydrogen nebula that will explain the appearance of the $2 \mathrm{I}-\mathrm{cm}$. profiles over this area of the sky. In Lawrence's paper, as in fact in Matthews' and Menon's papers, a preliminary investigation is made of the relation between the clouds that produce the optical interstellar absorption lines (clouds that may have very small angular dimensions) and the neutral hydrogen clouds that are observed by $2 \mathrm{I}-\mathrm{cm}$. technique (clouds which must have considerable angular dimensions to become detectable with present-day equipment).

Much of the current observational programme at the Agassiz Station deals with the section of the Milky Way between $l=33^{\circ}$ and $60^{\circ}$.

David S. Heeschen and Campbell M. Wade are studying the $2 \mathrm{I}-\mathrm{cm}$. profiles for intermediate latitudes $+10^{\circ},+15^{\circ}$ and $+20^{\circ}$ for positions $5^{\circ}$ apart in galactic longitude between $l=335^{\circ}$ and $50^{\circ}$. The primary aim of this research, which is well on the way toward completion, is to study the variation with galactic longitude of the widths, mean radial velocities and total intensities of the profiles, and also to investigate the presence of extended wings for some of the profiles. The variations in the $2 \mathrm{I}-\mathrm{cm}$. parameters are being correlated with the known optical features of the section, particularly with variations in the interstellar absorption.

A related programme calls for profiles at low galactic latitudes between $l=335^{\circ}$ and $l=35^{\circ}$; this programme is by David S. Heeschen and William E. Howard III. The primary purpose of this programme is to provide data for a preliminary, rather exploratory, study of the structure and motions of the inner regions of the Galaxy. In the first phase of this study, Heeschen and Howard are concentrating on the section between $l=335^{\circ}$ and $l=35^{\circ}$. This region contains a number of known $\mathrm{H}$ in regions and many stars with observed interstellar absorption lines. A preliminary analysis of our profiles corroborates the existence of a spiral arm interior to the Sagittarius arm, as found by Kwee, Muller and Westerhout [9].

To round out the research for this section of the Milky Way, May $\mathbf{K}$. Kassim is embarking on a programme for the section between $l=35^{\circ}$ and $60^{\circ}, b=-10^{\circ}$ and $+10^{\circ}$, at positions spaced $2^{\circ} 5$ apart. This research has the dual purpose of investigating the fine structure of the spiral features (curvature of arm, distribution of $\mathrm{H}$ I across the arm, expansion effects produced by $\mathrm{OB}$ aggregates) and of studying effects associated with the obscuration in the Great Rift.

The initial results obtained by Lawrence (paper 10) have encouraged Heeschen to initiate, jointly with Frank D. Drake, a programme for the 
study of possible correlation between $2 \mathrm{I}-\mathrm{cm}$. profiles and the interstellar lines observed by Adams [10]. Twenty-three centres, all but one of them outside the zone $b=-10^{\circ}$ to $+10^{\circ}$, have been selected in which there are two or more stars on Adams' list within the area covered by the antenna beam of our $24-\mathrm{ft}$. reflector. The average radial velocities and total intensities of the $2 \mathrm{I}-\mathrm{cm}$. profiles will be compared with the corresponding quantities for the interstellar absorption lines. The observations to date have indicated several regions of special interest. One of these is the region of the Lacerta aggregate, which has been mentioned by Westerhout (see paper 4). A second region, that of the Pleiades cluster, is now being investigated by Heeschen and Drake. They find that the $2 \mathrm{I}-\mathrm{cm}$. profile here is double-peaked; one peak has the radial velocity of the Pleiades cluster, while the radial velocity of the second peak corresponds to the mean radial velocity of the interstellar lines observed in this region.

Papers 8 (Matthews) and 9 (Menon) describe at length the programme for the section $l=60^{\circ}$ to $130^{\circ}$ and the Orion section. To supplement Matthews' programme, Robert J. Davis is observing positions separated by $2^{\circ}$ in galactic latitude for $b=-10^{\circ}$ to $+10^{\circ}$ at $l=120^{\circ}$ and also a strip at $b=+3^{\circ}$ with positions between $l=110^{\circ}$ and $l=130^{\circ}$ separated by $2^{\circ}$ in galactic latitude. At the request of George H. Herbig of Lick Observatory, Menon is studying the profiles for NGG 2244, NGG 2264 and surroundings.

Two features of the Agassiz Station programmes remain to be stressed. The first is that, with the completion of the above programmes, we shall have available high-resolution $2 \mathrm{I}-\mathrm{cm}$. profiles for most of this galactic belt between $l=335^{\circ}$ and $l=180^{\circ}$. It is our intention to publish in the not-toodistant future the derived mean profiles for all positions and thus make our material widely available for further analysis. The second feature of our present programme is that we consider it exploratory to the extent that we intend to observe all regions of special interest again and intensively with the 6o-ft. reflector and new electronic equipment now under construction.

Our present programme is limited to some extent by the nature of our electronic equipment. Our strength seems to lie in the very high frequency resolution provided by Ewen's equipment, which is in essence a very fine comparison radiometer. At the moment we are not equipped for studies of the continuous background, or for relatively broad-band research on galaxies or inter-galactic matter. We hope to enter research in these and related fields in the near future. 


\section{AGKNOWLEDGMENTS}

A project in radio astronomy, and notably in the $2 \mathrm{I}-\mathrm{cm}$. field, is expensive. The Agassiz Station project could not have been initiated without substantial support from a friend of the G. R. Agassiz Station and from the National Science Foundation. Continuing support from these same two sources, as well as through funds toward basic maintenance and operation provided by the Harvard Observatory Council, is proving to be essential for the operation of the programmes outlined above. The National Science Foundation has provided most of the funds for the construction of the $60-\mathrm{ft}$. reflector and a special gift from a friend accounts for the remainder. Two special gifts and a grant from the Research Corporation have given us the necessary funds for the construction of the new electronic equipment.

A successful project in $2 \mathrm{I}-\mathrm{cm}$. research requires more than funds alone. The enthusiastic encouragement which we have received from the organizations and individuals that have given us financial support and from the Observatory's Visiting Committee has been a constant source of inspiration. We have received much assistance already from some of the large electronic industries and without prospects of continued help of this nature we could hardly hope to remain successful. Our present group of reports provides in itself adequate proof that our relations with $D$. S. Kennedy and Company have been far more intimate than the average one between purchaser and manufacturer.

In concluding this introductory statement, it does not seem out of order for me to express my appreciation to my immediate associates in the Agassiz Station project. To Harold I. Ewen, who as co-director of the project remains a valued friend and advisor, goes the credit for the excellence of our equipment. To John A. Campbell, the project-engineer, my associates and students join me in expressing our heart-felt thanks for his continued and unceasing effort to keep the equipment in tip-top running condition. I cannot mention here all my students, but it would be unfair to close without a special word of thanks to A. Edward Lilley, now at the Naval Research Laboratory, and to David S. Heeschen, now back with us as the resident Agassiz Station Radio Astronomer; Heeschen and Lilley saw the project through its initial difficult period and we. all realize our indebtedness to them for their efforts on its behalf. 


\section{REFERENCES}

[1] Bok, B. J. J. Geophys. Res. 49, 192, I954.

[2] Heeschen, D. S. Ap. J. 121, 569, 1955.

[3] Lilley, A. E. Ap. J. 121, 559, 1955.

[4] Bok, B. J. and Ewen, H. I. A.J. 59, 318, 1954 .

[5] Bok, B. J. A.J. 6o, I46, I955.

[6] Heeschen, D. S. and Lilley, A. E. Proc. Nat. Acad. Sci. 40, ro95, 1954.

[7] Bok, B. J., Lawrence, R. S. and Menon, T. K. P.A.S.P. 67, 108, 1955.

[8] Matthews, T. A. P.A.S.P. 67, I 12, 1955.

[9] Kwee, K. K., Muller, G. A. and Westerhout, G. B.A.N. 12, 2 I I, no. 458, 1954.

[10] Adams, W. S. Ap. J. 1o9, 354, 1949. 